STOCHASTIC SYSTEMS

Theory and Applications 
This page is intentionally left blank

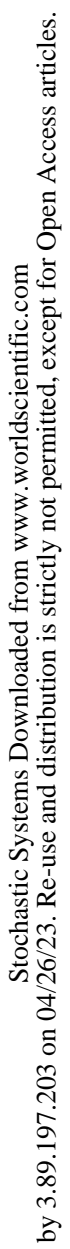




\section{STOCHASTIC SYSTEMS}

\section{Theory and Applications}

\section{S. Pugachev I. N. Sinitsyn \\ Russian Academy of Sciencies}

Translated by

I. V. Sinitsyna 


\section{Published by}

World Scientific Publishing Co. Pte. Ltd.

P O Box 128, Farrer Road, Singapore 912805

USA office: Suite 1B, 1060 Main Street, River Edge, NJ 07661

UK office: 57 Shelton Street, Covent Garden, London WC2H 9HE

\section{British Library Cataloguing-in-Publication Data}

A catalogue record for this book is available from the British Library.

\section{STOCHASTIC SYSTEMS \\ Theory and Applications}

Copyright $\odot 2001$ by World Scientific Publishing Co. Pte. Ltd.

All rights reserved. This book, or parts thereof, may not be reproduced in any form or by any means, electronic or mechanical, including photocopying, recording or any information storage and retrieval system now known or to be invented, without written permission from the Publisher.

For photocopying of material in this volume, please pay a copying fee through the Copyright Clearance Center, Inc., 222 Rosewood Drive, Danvers, MA 01923, USA. In this case permission to photocopy is not required from the publisher.

ISBN $981-02-4742-7$ 


\section{PREFACE}

The monograph is dedicated to the systematic presentation of the applied advanced theory and analytical methods for the stochastic systems, i.e. the dynamical systems described by the finite- and infinitedimensional stochastic differential, difference, integral, integrodifferential etc. equations. It is based on the results of the fundamental research performed in the Institute for Informatic Problems of the Russian Academy of Sciences in the context of the scientific program "Stochastic Systems" and the lecture courses delivered by the authors in the domestic and the foreign Technical Universities.

The book may be used as the textbook for the applied mathematics faculties of the universities. The unified procedure, thorough selection of the examples and the problems (over 500) and the large applications make this book useful for the graduates, the post-graduates and the lecturers. The book is of considerable interest also for the mathematicians who deal with the stochastic equations and their applications.

The construction of general stochastic systems theory is based on the equations for the multi-dimensional characteristic functions and functionals. The stochastic differential equations with the arbitrary processes with the independent increments are studied. The equations with the Wiener and the Poisson processes are considered as a special case. The methods of the parametrization of the multi-dimensional distributions in the nonlinear stochastic systems based on the moments, the semiinvariants, the quasimoments and on the consistent orthogonal expansions are stated systematically. Special attention is paid to linear and reducible to linear stochastic systems theory based on the canonical representations (canonical expansions and integral canonical representations). Most attention has been concentrated on the structural theory of the composed stochastic systems on the grounds of the conditional probability measures.

Chapter 1 is devoted to the mathematical models of the dynamic systems under conditions of the random disturbances and their characteristics. The linear and the nonlinear continuous, discrete and continuousdiscrete systems described by the stochastic equations in the finite- and the infinite-dimensional spaces are considered. Special attention is paid to the composed stochastic systems.

The main notions of probability distributions theory of the random variables, the random processes and the random functions are stated in Chapter 2. After determining the probability spaces, the conditional 
probabilities, probabilities in the finite and the infinite products of the spaces are considered. The conditions of the existence of the regular probabilities are established. Further the different probability measures of the random functions and the probabilities of events connected with the random functions are studied in detail. The last sections are devoted to the distributions of the separable random functions, to the derivation of the criteria of the continuity and the differentiability of the random functions.

Chapter 3 contains the account of the basic notions connected with the expectation of the random variables in Banach and topological linear spaces, the properties of the operators of the moments of the second and higher orders, the properties of the characteristic functionals and the correspondence between the probability measures and the characteristic functionals are established. The conditional operators of the moments and their properties are studied in detail. Special section is devoted to the elements of the probabilities distribution in the infinite-dimensional spaces. The properties of the normal distribution on the product of two spaces, the conditional normal distributions and the normal distributions in Hilbert spaces are considered. Approximate representation of the oneand the multi-dimensional distributions based on the consistent orthogonal expansions is treated. Special attention is paid to the semiinvariant and the quasimoments. A systemical presentation of general theory of the canonical expansions is given. Practical ways of the canonical expansions constructing are described. The joint canonical expansions are considered.

Chapter 4 is devoted to the selected topics of stochastic calculus. The theory of the convergence, the continuity and the differentiability is considered. The integrability of the random functions over nonrandom and random measures is discussed. The theory of the stochastic Itô integrals, the symmetrized (Stratonovich) integral and the $\theta$-integral is outlined systematically. The stochastic integrals over the Wiener and the Poisson measures are studied. The connection between the processes with the independent increments and the Ito integral is established. The elements of the theory of the generalized random functions are stated. A systematic account to the integral canonical representations is given. The connection of the integral canonical representations with the spectral one is established. Practical ways of constructing the integral canonical representations and, in particular, the methods of the shaping filters are considered. The joint integral canonical representations are studied. 
The general theory of the finite- and the infinite-dimensional stochastic systems is given in Chapter 5. At the beginning the differentiation of the Itô process is set out in detail. The Itô formulae for the differentiation of the composite functions in the cases of the Wiener, the Poisson and the general random process with the independent increments have a significant place. The theory of the stochastic integral, differential and integrodifferential Itô equations including the methods of reducing the state equations to the stochastic differential equations, change of variables, sufficient conditions of the existence and the uniqueness of the mean square solutions are considered. The central problem of stochastic system theory - the problem of calculation of the multidimensional distributions is stated. The elements of numerical integration of the stochastic differential equations are given. The theory of the one- and the multi-dimensional distributions in the continuous, discrete and continuous-discrete systems in the finite-dimensional spaces are stated in detail. For such systems the known equations (Fokker-PlankKolmogorov, Feller--Kolmogorov, Pugachev and their generalizations) are derived. The problems of one-dimensional and multi-dimensional distributions theory on the basis of the equations for the correspondent characteristic functionals in composite stochastic systems including the stochastic systems in the Hilbert spaces are considered. The main statements of the structural theory of the composed stochastic systems on the basis of conditionally probability measures are outlined. The last section contains the applications to optimal online filtering problems. Simple derivation of the Kushner-Stratonovich and the Zakai equations for the normal white noises is presented. Corresponding equations for the composite stochastic systems are given in Problems.

Chapter 6 contains the account of the main methods of linear stochastic systems theory in connection with continuous, discrete and continuous-discrete systems. The spectral and correlation methods, the methods of general theory of linear stochastic systems are outlined. The correlation methods based on the canonical representations are considered with great attention. The general theory of the canonical representations of the linear transformations is given. The separate subsections are devoted to the solution of the linear operator equations by the canonical representations methods. The applications to the offline spectral and correlation analysis and the shaping linear filters design are given. Software for the analytical modeling problems is described. The Kalman-Bucy and the Liptser-Shiryaev online filters are considered. 
Chapter 7 is devoted to the approximate methods of nonlinear finite- and infinite-dimensional stochastic systems theory based on the parametrization of the distributions. The normal approximation, statistical and the equivalent linearization methods are considered. The normalization of the composite stochastic systems is treated. General problem of parametrization of the multi-dimensional distributions is stated. The methods of the moments, the seminvariants, the quasimoments and the orthogonal expansions are given. The practical problems of reducing the number of equations for the automatic derivation of the equations and the corresponding software for distribution parameters are considered. The solution of the structural parametrization problem of the multi-dimensional distributions is outlined on the basis of the ellipsoidal approximation method. The canonical representations methods for the nonlinear stochastic systems are considered. The basic methods are given for stochastic systems with the normal white noises. Corresponding methods for the composite stochastic systems are given in Problems. The applications of the given methods to the nonlinear stochastic systems offline and online analysis, modeling and filtering problems in mechanics, physics, biology, chemistry, finance and control are stated. Specialized software is described.

Chapters 1, 5-7 give efficient toolkit for the analytical modeling and the design of the stochastic systems. To facilitate the mastering of the stated methods about 500 thoroughly selected examples and problems are given in the correspondent subsections, sections and chapters of this book.

Appendices 1-7 contain auxiliary materials which include the information about tables of stochastic Itô differentials for the typical composite functions, the Hermite polynomials of a vector argument, the polynomials orthogonal to gamma and $\chi^{2}$-distributions, the tables of the coefficients of statistical linearization for the typical composite functions, integrands evaluations in the equations of the basic methods of distribution parametrization, the tables of a basic characteristics and transformation the structural rules of the linear systems and the tables of some definite integrals and special functions.

For information about the notions and theorems from vatious parts of mathematics used in the book we advise (Korn and Korn 1968).

Bibliographical notes and References are given at the end of the book. The authors do not pretend in any way to provide a complete bibliography. Only those sources are given in the list which are cited in the text. Basic notations are presentented before Index. 
The book is divided into chapters, sections and subsections. The formulae (examples) are numerated with indication of the number of the chapter and the section, for instance, (1.2.3) means that formula (3) belongs to section 2 of chapter 1 . The problems are numerated only with indication of the number of chapter. At each Appendix the separate numeration is accepted.

For the convenience of the readers the formulations of all basic results and statements are given in the separate theorems and the corollaries or in the text italics. The beginnings and the ends of the evaluations, the proofs and the discussions which lead to certain results are indicated by triangular indices $\triangleright$ and $\triangleleft$.

The book may be used for studying stochastic systems theory by the different categories of the readers. When reading this book it is necessary to know the foundations of probability theory, for instance, the book (Pugachev 1984) and the basis of functional analysis, for instance, (Pugachev and Sinitsyn 1999). A reader who is the master of the foundations of the random functions theory and the stochastic differential equations may study directly Chapters 5-7 after Chapter 1 . Finally, for studying the finite-dimensional stochastic systems theory in the simplest statement it is sufficient to study Chapter 1 (Sections 1.1-1.5), Chapter 2 (Sections 2.1.-2.10), Chapter 3 (Sections 3.1, 3.3-3.5, 3.9), Chapters 4-7 (excluding the subsections concerning the infinite-dimensional systems).

The book may be used for the lecturers on probability theory and stochastic calculus, on statistical foundations of system analysis, information, radioengineering, control and communication theory, reliability theory of structures, dynamics of machines and mechanisms, and some fragments in specialized courses, for example, analytical mechanics of stochastic systems, statistical theory of oscillations and stability and etc.

Systematical statement of the methods of the canonical expansions theory and the integral canonical representations in the finite- and the infinite-dimensional spaces and their applications are given in the book for the first time (Sections 3.9, 4.8, 6.3, 6.4, 7.7). This material may be used for the special courses on applied mathematics. Many examples and problems are original, have complex character and therefore may be used for the students and the post-graduates works.

The suggestion that the authors could write a series of book for World Scientific was put forward by Editor Dr. Sen Hu in 1998. Dr. Jitan Lu performed the final work as desk editor during 2001. The authors are particularly indebted to them. 
The authors are very much obliged to Mrs. I.V.Sinitsyna for her translation of the manuscript and the list of References preparation, Mrs. E.N.Fedotova for the camera ready copy of the manuscript preparation, Mrs. I.V.Makarenkova and Mr. V.V.Belousov for the figures preparation.

The authors also owe thanks to M.E.Shaikin for his valuable remarks concerning the book as a whole, to A.V.Bosov, A.V.Borisov, B.G.Dostupov, P.S.Igumnov, I.E.Kazakov, E.R.Korepanov, V.N.Pugachev, V.I.Shin, V.I.Sinitsyn, M.I.Tleubergenov whose help considerably improved the manuscript.

The authors acknowledges the help of our colleagues in Russia and abroad. We are also grateful to the Institute for Informatics Problems, the Russian Academy of Sciences, the Russian Foundation of Fundamental Research, the International Institute for Applied Systems Analysis (Austria, Laxenburg), the Delft Technical University (The Netherlands), the Institute of Thermodynamics (Czech Republic) for their support.

Moscow

1997-2001
V.S.Pugachev

I.N.Sinitsyn

Institute for Informatics Problems Russian Academy of Sciences 


\section{CONTENTS}

Preface $\ldots \ldots \ldots \ldots \ldots \ldots \ldots \ldots \ldots \ldots \ldots \ldots \ldots, \mathbf{v}$

Chapter 1. Dynamical Systems and Their Characteristics.. 1

1.1. Continuous Systems $\ldots \ldots \ldots \ldots \ldots \ldots \ldots \ldots \ldots \ldots \ldots \ldots$

1.1.1. Mathematical Models of Continuous Systems (1), 1.1.2. Characteristics of Continuous Systems (7). 1.1.3. Weighting Function of One-Dimensional Linear System (11). 1.1.4. Stability of Linear Systems (15). 1.1.5. Stationary Systems (17). 1.1.6. Transfer Function of Stationary Linear System (17). 1.1.7. Frequency Response of Stationary Linear System (19).

1.2. Linear Differential Systems $\ldots \ldots \ldots \ldots \ldots \ldots \ldots \ldots \ldots \ldots \ldots \ldots$

1.2.1. Equations of Linear System and Its Weighting Function (23). 1.2.2. Finding Weighting Function by Adjoint System Method (26). 1.2.3. Transformation of Linear System Equations into Cauchy Form (27), 1.2.4. Inverse Systems (32).

1.2.5. Transfer Function of Stationary Linear System (37).

1.3. Connections of Systems

1.3.1. Basic Systems Connections (42). 1.3.2. Weighting Functions of Linear Systems Typical Connections (45). 1.3.3. Connections of Stationary Linear Systems (49). 1.3.4. Structural Transformation Rules (52).

1.4. Stochastic Differential Systems $\ldots \ldots \ldots \ldots \ldots \ldots \ldots \ldots \ldots .55$

1.4.1. General Form of Equations of Stochastic Differential Systems (55). 1.4.2. Stochastic Linear Differential Systerns (57). 1.4.3. Linear Systems with Parametric Noises (57). 1.4.4. Stochastic Bilinear Differential Systems (58).

1.5. Stochastic Discrete Systems ......................

1.5.1. Discrete Systems (59). 1.5.2. Characteristics of Discrete Linear Systems (60). 1.5.3. Discrete Linear Stationary Systems (66). 1.5.4. Discrete Stationary Systems Described by Difference Equations (72). 1.5.5. Discrete Linear Systems Described by Difference Equations (75). 1.5.6. Discrete Nonlinear Systems Described by Difference Equations (78). 1.5.7. Stochastic Discrete Linear and Nonlinear Systems (79).

1.6. Stochastic Composed Systems. Basic Problems of Stochastic

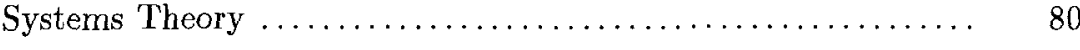

1.6.1. Definitions (80). 1.6.2. Main Types of Stochastic Systems Connections (81). 1.6.3. Stochastic Systems with Distributed Parameters (86). 1.6.4. Stochastic Composed Systems (86). 1.6.5. Problems of Stochastic Systems Theory (87). 


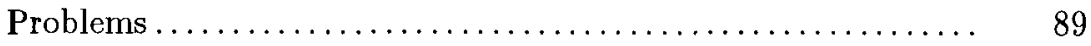

Chapter 2. Random Variables, Processes and Functions $\quad 97$

2.1. Probability Spaces ........................... 97

2.1.1. Space of Elementary Events (97). 2.1.2. Probability Spaces (99).

2.2. Random Variables ........................... 102

2.2.1. Definitions (102) 2.2.2. Probability Measure (103). 2.2.3. Equivalence of

Random Variables (104). 2.2.4. Two Types of Convergence of Random Sequences

(105). 2.2.5. Finite-Dimensional Random Variables (107).

2.3. Conditional Probabilities $\ldots \ldots \ldots \ldots \ldots \ldots \ldots \ldots \ldots . . . \ldots \ldots$

2.3.1. Definitions (110). 2.3.2. Properties of Conditional Probability (111).

2.3.3. Regular Conditional Probability (112). 2.3.4. Conditional Distribution of Random Variable (113)

2.4. Probabilities in Finite Product of Spaces ............. 114

2.4.1. Definitions (114). 2.4.2. Probability in Two Spaces Product (115).

2.4.3. Calculation of Probability in Finite Product of Spaces (121). 2.4.4. Independence of Random Variables (123).

2.5. Probabilities in Infinite Product of Spaces ........... 125 2.5.1. Introductory Remarks (125). 2.5.2. Distributions in Infinite Product of Spaces (126)

2.6. Existence of Regular Conditional Probabilities ......... 130 2.6.1. Definitions (130). 2.6.2. Kolmogorov Theorem and its Generalizations (131)

2.7. Probability Measure of Random Function

2.7.1. Definitions (133). 2.7.2. Measurable Random Functions (135). 2.7.3. Multi-Dimensional Distributions of Random Function (135). 2.7.4. Markov Random Processes (138). 2.7.5. Normal Random Processes (140). 2.7.6. Conditionally Normal Random Processes (141). 2.7.7. Streams of Events (142). 2.7.8. Excursions of Random Functions (145). 2.7.9. Probabilities of Events Connected with Random Functions (146).

2.8. Separable Random Functions

2.8.1. Definitions (149), 2.8.2. Multi-Dimensional Distributions for Separable Random Function (150). 2.8.3. Separable Random Functions and Their Realizations (152) 2.8.4. Equivalent Random Functions and Their Realizations (155).

2.9. Continuous Random Functions

2.9.1. Definitions (157). 2.9.2. Separant for Continuous Random Function (158).

2.9.3. Measurability and Separability of Continuous Random Function (159). 2.9.4. Sufficient Criteria of Continuity (161). 
2.10. Differentiable Random Functions $\ldots \ldots \ldots \ldots \ldots \ldots \ldots . \quad 167$

2.10.1. Definitions (167) 2.10.2. Sufficient Conditions of Differentiability (168).

2.10.3. Trace Probabilities (173).

Problems.................................... 176

Chapter 3. Moments, Characteristic Functions,

Functionals and Canonical Expansions ............ 179

3.1. Expectation of Random Function ................ 179

3.1.1. Definitions (179), 3.1.2. Properties of Expectation (182).

3.2. Expectation of Random Variable in Topological Linear

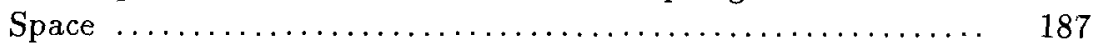

3.2.1. Definitions (187) 3.2.2. Properties of Expectations (189).

3.3. Moments ................................. 190

3.3.1. Definitions (190). 3.3.2. Properties of Second Order Moments (197). 3.3.3.

White Noise (205). 3.3.4. Moments of Higher Orders (206).

3.4. Conditional Moments ........................ 209

3.4.1. Definitions (209). 3.4.2. Properties of Conditional Moments (211).

3.4.3. Properties of Conditional Moments Operators (218)

3.5. Characteristic Functions and Functionals ............ 218

3.5.1. Definitions (218). 3.5.2. Properties of Characteristic Functional (221).

3.5.3. Relation between Characteristic Function and Moments (223).

3.5.4. Expansion of Characteristic Functional (224). 3.5.5. Correspondence between Probability Measures and Characteristic Functionals (225).

3.6. Sequences of Probability Measures and Characteristic

Functionals. Distributions in Banach Spaces .............. 230

3.6.1. Helly-Bray Theorem (230). 3.6.2. Direct and Inverse Levi Theorems (231).

3.6.3. Finite-Dimensional Case of Bochner Theorem (234). 3.6.4. General Case of Bochner Theorem (236). 3.6.5. Probability Distributions in Banach Spaces. Mourier Theorem (241).

3.7. Normal Distributions in Linear Spaces ............. 246

3.7.1. Properties of Normal Distribution (246). 3.7.2. Normal Distribution on Product of Two Spaces (248). 3.7.3. Conditional Distributions (250). 3.7.4. Normal Distribution in $H$-space (258).

3.8. Approximate Representation of Distributions .......... 266

3.8.1. Pearson Curves System (266). 3.8.2. Orthogonal Expansion of Density (266). 3.8.3. Hermite Polynomial Expansion of Density (273). 3.8.4. Relations between Quasimoments and Semiinvariants (274). 3.8.5. Edgeworth Series (276). 
3.8.6. Consistent Biorthogonal Systems of Polynomials (279). 3.8.7. Consistent Orthogonal Expansions of Multi-Dimensional Densities (281). 3.8.8. Consistent Hermite Polynomial Expansions of Multi-Dimensional Densities (283).

3.9. Canonical Expansions ......................... 284 3.9.1. Introductory Remarks (284). 3.9.2. Auxiliary $H$-space (286). 3.9.3. Direct Theorem about Canonical Expansions (291). 3.9.4. Inverse Theorem about Canonical Expansions (298). 3.9.5. Way to Construct Canonical Expansions (299). 3.9.6. Canonical Expansion of Random Variable in $H$-space (300) . 3.9.7. Joint Canonical Expansion of Two Random Variables (303).

Problems .......................................

Chapter 4. Stochastic Integrals, Spectral and Integral

Canonical Representations ....................... $\quad \mathbf{3 1 9}$

4.1. Mean Square Limits ........................... $\quad 319$

4.1.1. Definitions (319). 4.1.2. Theorems about m.s. Convergence (322).

4.1.3. Theorems about m.s. Convergence of Random Function in Linear Space (325).

4.2. Mean Square Continuity of Random Function ......... 326

4.2.1. Definitions (326). 4.2.2. Necessary and Sufficient Conditions of m.s. Continuity (327). 4.2.3. Processes with Uncorrelated Increments (328).

4.3. Differentiation of Random Functions

4.3.1. Definitions (330). 4.3.2. Theorems about m.s. Differentiability (330).

4.3.3. Theorems about m.s. Differentiability of Random Function in Linear Space (334).

4.4. Integration of Random Function over Nonrandom Measure

4.4.1. Definitions (336). 4.4.2. Theorems about m.s. $\mu$-integrability of Random Function (338).

4.5. Integration over Random Measure $\ldots . \ldots \ldots \ldots \ldots \ldots \ldots . \quad 343$

4.5.1. Definitions (343). 4.5.2. Necessary and Sufficient Conditions of Integrability over Random Measure (344).

4.6. Stochastic Integral .......................... 345

4.6.1. Definitions (345). 4.6.2. Conditions of Existence of Stochastic Integral (349). 4.6.3. Processes with Independent Increments (350). 4.6.4. White Noise in Strict Sense (355). 4.6.5. Wiener Processes and Measures (355). 4.6.6. Poisson Processes and Measures (357). 4.6.7. Itô Stochastic Integral (358). 4.6.8. Stochastic Integrals over Wiener and Poisson Measures (361). 4.6.9. Other Types of Stochastic Integrals (365). 
4.7. Generalized Random Functions $\ldots \ldots \ldots \ldots \ldots \ldots \ldots \ldots . \quad 366$ 4.7.1. Definitions (366), 4.7.2. While Noise as Generalized Function (368). 4.7.3. Convergence of Generalized Random Functions (369). 4.7.4. Differentiation of Generalized Random Functions (371). 4.7.5. Integration of Generalized Functions (373). 4.7.6. Stochastic Integrals as Integrals Containing White Noise (375).

4.8. Integral Canonical and Spectral Representations ....... 376 4.8.1. Introductory Remarks (376). 4.8.2. Direct Theorem about Integral Canonical Representations (378). 4.8.3. Inverse Theorem about Integral Canonical Representations (382). 4.8.4. Integral Canonical Representations and Shaping Filters (387). 4.8.5. Joint Integral Canonical Representation of Two Random Variables (388). 4.8.6. Spectral and Integral Canonical Representations of Scalar Random Functions (392). 4.8.7. Spectral and Integral Canonical Representations of Vector Stationary Random Function (396). 4.8.8. Way of Constructing Integral Canonical Representation of Vector Stationary Random Fucntion (397).

Problems

\section{Chapter 5. General Theory of Stochastic Systems and}

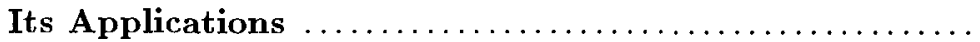

5.1. Stochastic Differentials ....................... 425

5.1.1. Stochastic Differential of Itô Process (425). 5.1.2. Itô Formula (427).

5.1.3. Generalized Itô Formulae (432). 5.1.4. Other Types of Stochastic Differentials (438).

5.2. Stochastic Differential Equations $\ldots \ldots \ldots \ldots \ldots \ldots \ldots \ldots, 442$

5.2.1. Definitions (442) 5.2.2. Stochastic Differential Equation Determines Markov Process (444). 5.2.3. Change of Variables in Stochastic Differential Equation (444). 5.2.4. Other Types of Stochastic Differential Equations (447). 5.2.5. Transformation of Stochastic Differential Equation into Itô Equation (447). 5.2.6. Sufficient Conditions of Existence and Uniqueness of m.s. Solutions of Stochastic Differential Equation (450).

5.3. Differential Equations Containing Random Functions ......

5.3.1. Definitions (453) 5.3.2. Sufficient Conditions of Existence and Uniqueness of Solutions of Differential Equations Containing Random Functions (455).

\subsection{Transformation of System Equations into Stochastic}

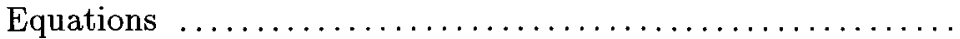

5.4.1. Principal Possibility of Replacing Random Function in Differential Equation by White Noise (459). 5.4.2. Method of Shaping Filters (460). 5.4.3. Equations of Stochastic Differential Systems. Central Problem of Stochastic Systems Theory (461). 
5.5. Numerical Integration of Stochastic Differential Equation .

5.5.1. Introductory Remarks (463). 5.5.2. Existence of Exact Difference Equation Correspondent to Given Stochastic Differential Equation (465). 5.5.3. Euler Method (466). 5.5.4. More Exact Equations (467). 5.5.5. Distribution of Random Vector $V_{n}$ (474). 5.5.6. Analysis of More Precise Equations (476). 5.5.7. Strong and Weak Approximation of Nonlinear Stochastic Differential Equations (478).

\subsection{Multi-Dimensional Distributions in Stochastic Differential}

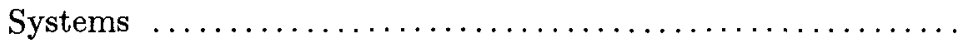

5.6.1. One-Dimensional Characteristic Function (479). 5.6.2. Multi-Dimensional Characteristic Functions (483). 5.6.3. Specific Form of Equation for Characteristic Functions (484). 5.6.4. Equations for Multi-Dimensional Densities (486). 5.6.5. Formulae for Function $\chi(\mu ; t)$ (487). 5.6.6. Equations for Multi-Dimensional Densities in Case of Wiener Process (489). 5.6.7. Equation for Transition Density (493). 5.6.8. Case $a(y, t)$ Polynomial in $y$ and $b(y, t)$ Independent on $y$ (494). 5.6.9. Case of $a(y, t)$ Polynomial and Normal White Noise (495). 5.6.10. Multi-Dimensional Distributions in Stochastic Continuous Composite Systems (497). 5.6.11. Stationary Processes in Stochastic Differential Systems (498). 5.6.12. Sufficient Conditions of Existence of Multi-Dimensional Distributions (501).

5.7. Multi-Dimensional Distributions in Stochastic Discrete and Continuous-Discrete Systems ..................... 506

5.7.1. Multi-Dimensional Distributions in Stochastic Discrete Systems (506). 5.7.2. Distributions in Stochastic Continuous-Discrete Systems

5.8. Distributions in Stochastic Composed Systems .......... 5.8.1. Structural Theory of Stochastic Composed Systems (510). 5.8.2. Distributions in Stochastic Systems with Random Structure (512).

5.9. Distributions in Stochastic Infinite-Dimensional Systems .

5.9.1. Distributions in Stochastic Continuous Hilbert Systems (518). 5.9.2. Distributions in Markov Stochastic Discrete Systems (522).

5.10. Applications. Optimal Online Filtering $\ldots \ldots \ldots \ldots \ldots$

5.10.1. Introductory Remarks (523). 5.10.2. General Formula for Optimal Filtering (525). 5.10.3. Auxiliary Problem (526). 5.10.4. Equation for Conditional Characteristic Function (534). 5.10.5. Equation for Conditional Density (535). 5.10.6. Equations for Nonnormalized Conditional Density and Characteristic Function (536). 5.10.7. Stochastic Differential of Conditional Expectation (539). 5.10.8. Stochastic Differential of Conditional Second Order Moment (539). 5.10.9. Stochastic Differential of Conditional Covariance Matrix (541). 5.10.10. Optimal Filtering in Stochastic Discrete Systems (542). 
Problems ........................................

\section{Chapter 6. Methods of Linear Stochastic Systems Theory} and Their Applications

6.1. Spectral and Correlation Methods ................... 557

6.1.1. Representation of State Vector (557). 6.1.2. Representations of First and Second Order Moments (558), 6.1.3. Differential Equation for Expectation (558). 6.1.4. Differential Equation for Covariance Matrix (559). 6.1.5. Differential Equation for Second Order Moment (560) 6.1.6. Differential Equation for Covariance Function (561) 6.1.7. Stationary Processes in Stationary Linear Systems (563). 6.1.8. Spectral Methods (565). 6.1.9. Spectral and Correlation Methods for Stochastic Discrete Linear Systems (569), 6.110. Correlation Theory of Linear Transformations (573). 6.1.11. Spectral and Correlation Methods for Stochastic Infinite-Dimensional Linear Systerns (573).

6.2. Methods of General Linear Stochastic Systems Theory .... 575 6.2.1. Equations for Characteristic Functions (575). 6.2.2. Integration of Equations for Characteristic Functions (576). 6.2.3. Explicit Formulae for MultiDimensional Characteristic Functions (570). 6.2.4. Case of Normal Distribution of System State (585). 6.2.5. Strictly Stationary Processes in Stationary Linear Systems (588). 6.2.6. Methods of General Theory of Stochastic Discrete Linear Systems (590), 6.2.7. Methods of Stochastic Infinite-Dimensional Linear Systems Theory (591).

6.3. Methods of Linear Theory of Stochastic Systems based on Canonical Expansions ........................... $\quad 592$ 6.3.1. Introductory Remarks (592). 6.3.2. Theory of General Linear Transfomations (595). 6.3.3. Assignment of Probability Measures of Canonical Expansions (601). 6.3.4. Solution of Equations by Canonical Expansions (605).

6.4. Methods of Linear of Stochastic Systems Theory based on Integral Canonical Representations $\ldots \ldots \ldots \ldots \ldots \ldots \ldots \ldots$.

6.4.1. Introductory Remarks (613). 6.4.2. General Theory of Integral Canonical Representations of Linear Transformations (615). 6.4.3. Solution of Equations by Integral Canonical Representations Method (618).

6.5. Applications. Shaping Filters Design and Modeling .......

6.5.1. Shaping Filters for Scalar Stationary Processes (624). 6.5.2. Shaping Filter for Vector Stationary Process (631). 6.5.3. Shaping Filter for Process Reducible to Stationary One (632) 6.5.4. Shaping Filters for Stationary Functions of Vector Argument (634), 6.5.5. Software for Analytical Modeling Problems (639). 
6.6.1. Equations of Linear Filtering (641). 6.6.2. Kalman-Bucy Filter (643).

6.6.3. Case of Equations Linear in State Vector (645).

Problems ......................................

\section{Chapter 7. Methods of Nonlinear Stochastic Systems} Theory and Their Applications

7.1. Moments of State Vector $\ldots \ldots \ldots \ldots \ldots \ldots \ldots \ldots \ldots \ldots \ldots \ldots$

7.1.1. Formula for Derivative of Expectation (661). 7.1.2. Formula for Derivative of Second Order Moment (662), 7.1.3. Formula for Derivative of Covariance Matrix (663), 7.1.4. Formulae for Derivatives of Second Order Moment and Covariance Function (664). 7.1.5. Infinite Set of Equations for Moments (665). 7.1.6. Linear Systems with Parametric Noises (667). 7.1.7. Formulae for Moments in Stochastic Discrete Nonlinear Systems (671). 7.1.8. Equations for Moments of Discrete Linear System with Parametric Noises (671).

\subsection{Methods of Normal Approximation and Statistical}

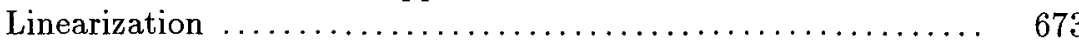

7.2.1. Normal Approximations Method. One-Dimensional Distribution (673). 7.2.2. Statistical Linearization Method (674). 7.2.3. Normal Approximation Method. Multi-Dimensional Distributions (680). 7.2.4. Approximate Determination of Stationary Processes (683). 7.2.5. Normal Approximation Method for Stochastic Discrete Nonlinear Systems (684). 7.2.6. Normal Approximation Method for Stochastic Infinite-Dimensional Nonlinear Systems (685). 7.2.7. Methods of Equivalent Linearization and Structural Methods (687). 7.2.8. General Problem of Stochastic Systems Normalization (690).

7.3. Methods of General Nonlinear Stochastic Systems Theory.

Parametrization of Multi-Dimensional Distributions .......... 691

7.3.1. Introductory Remarks (691). 7.3.2. General Problem of Multi-Dimensional Distributions Parametrization (692). 7.3.4. Reducing Number of Equations for Distribution Parameters (693).

7.4. Methods of Moments and Semiinvariants ............ 695

7.4.1. One-Dimensional Distribution. Initial Moments (695). 7.4.2. One-Dimensional Distribution. Central Moments (697). 7.4.3. Multi-Dimensional Distributions. Initial Moments (704). 7.4.4. Multi-Dimensional Distributions. Central Moments (706). 7.4.5. Approximate Determination of Stationary Processes (711). 7.4.6. Moments Method for Stochastic Discrete Nonlinear Systems (712). 7.4.7. Moments Method for Stochastic Infinite-Dimensional Systems (713). 7.4.8. Semiinvariant and Moment-Semiinvariant Methods (714). 
7.5. Methods based on Orthogonal Expansions and Quasimoments 7.5.1. Orthogonal Expansion of One-Dimensional Distribution (719). 7.5.2. Method of Quasimoments (722). 7.5.3. Consistent Orthogonal Expansions of MultiDimensional Distributions (724). 7.5.4. Consistent Hermite Polynomial Expansions (728). 7.5.5. Approximate Determination of Stationary Processes (732). 7.5.6. Reducing Number of Equations (733). 7.5.7. Methods based on Orthogonal Expansions for Discrete Nonlinear Systems (736). 7.5.8. Other Methods of Distributions Parametrization (737).

7.6. Structural Parametrization of Distributions. Ellipsoidal

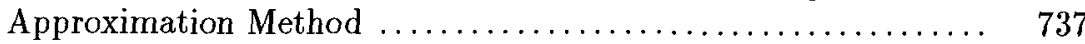

7.6.1. Introductory Remarks (737). 7.6.2. Ellipsoidal Approximation of OneDimensional Distribution (743). 7.6.3. Ellipsoidal Approximation of MultiDimensional Distributions (752). 7.6.4. Moments of State Vector (758). 7.6.5. EAM Accuracy (759).

7.7. Methods based on Canonical Representations .......... 768 7.7.1. Linearization by Canonical Expansions (768). 7.7.2. Linearization by Integral Canonical Representations (774). 7.7.3. Canonical Representations of Nonlinear Transformations Reducible to Linear Ones (776). 7.7.4. General Methods of Stochastic Nonlinear Systems Theory based on Canonical Representations (780).

\subsection{Applications. Analysis of Stochastic Systems and Analytical}

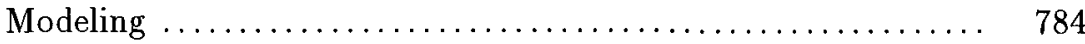

7.8.1. Accuracy Analysis of Single Loop Nonlinear Control Systems (784). 7.8.2. Solid Dynamics in Stochastic Media (788). 7.8.3. Stochastic Hereditary Systems (792). 7.8.4. Normalization of Risk and Profit Financial Transactions (799). 7.8.5. Normalization of Queueing Systems (799). 7.8.6. Software for Nonlinear Stochastic Systems Analytical Modeling (809).

\subsection{Online Conditionally Optimal Nonlinear Filltering .........}

7.9.1. Preliminary Remarks (813). 7.9.2. Equations Determining Optimal Filter Coefficients (815). 7.9.3. Estimation of Filtering Accuracy (817). 7.9.4. Generalized Kalman Filter (817). 7.9.5. Software for Conditionally Optimal Discrete Filter Design (821).

Problems

1. Stochastic Itô Differentials of Typical Composite Functions (837). 2. Hermite Polynomials. Polynomials Orthogonal to Gamma and $\chi^{2}$-Distibutions (839). 3. Statistical Linearization of Typical Composite Functions (853). 4. Integrands Evaluation in Equations of Methods based on Parametrization Distributions 
(856). 5. Basic Characteristics of Some Continuous Typical Linear Systems (864). 6. Structural Transformation Rules (870). 7. Some Definite Integrals and Special Functions (876).

Bibliographical Notes ........................ $\quad \mathbf{8 7 9}$

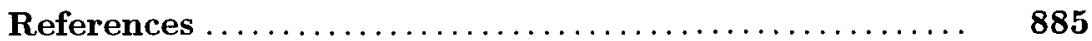

Notations $\ldots \ldots \ldots \ldots \ldots \ldots \ldots \ldots \ldots \ldots \ldots \ldots \ldots \ldots, \quad \mathbf{8 9 9}$

Index $\ldots \ldots \ldots \ldots \ldots \ldots \ldots \ldots \ldots \ldots \ldots \ldots \ldots \ldots, \quad 901$ 